Revista Signos

2010, 43(74)

$465-488$

\title{
Escritura académica a lo largo de la carrera: Un programa institucional
}

\author{
Estela Moyano \\ Universidad Nacional de General Sarmiento \\ Argentina
}

Resumen: La enseñanza de la lectura y la escritura, basada en el concepto de género en el nivel superior, se propone como objetivo promover el desarrollo de los estudiantes en el uso del lenguaje propio de ámbitos institucionales como la academia, la ciencia y los espacios de actuación profesional. Desde la perspectiva teórica seleccionada (Lingüística Sistémico-Funcional), en este artículo se presenta un programa para el desarrollo de habilidades de lectura y escritura a lo largo de la carrera universitaria basado en la teoría de género y registro. El programa, que cuenta con un grado alto de institucionalización, ha sido implementado en el segundo ciclo de las carreras y exige el compromiso de actores de diferente jerarquía institucional y diferentes especialidades disciplinares, además de los docentes en ciencias del lenguaje.

Palabras Clave: Lectura y escritura, universidad, Lingüística Sistémico-Funcional, escritura académica.

Recibido: 30-VI-2009 Aceptado: 20-IV-2010
Correspondencia: Estela Moyano (estelaimoyano@gmail.com). Universidad Nacional de General Sarmiento. Juan M. Gutiérrez entre José L. Suárez y Verdi 1613, B1613GSX, Los Polvorines, Buenos Aires, Argentina. 


\title{
Academic literacy across the university curriculum: An institutional program
}

\begin{abstract}
A genre-based pedagogic model for teaching literacy at tertiary level of education aims to promote students' literacy in institutional environments. From the theoretical perspective selected (Systemic-Functional Linguistics), a genre-based pedagogy influences knowledge construction in disciplines and empowers students to engage academic, scientific and professional social activities. This paper outlines a program for teaching literacy across the second cycle of university curriculum, the negotiation between participants as a critical tool and a brief evaluation. This institutional program has been implemented with a high degree of commitment of its actors -university authorities, professors of different disciplines and language teachers.
\end{abstract}

Key Words: Literacy, tertiary education, Systemic-Functional Linguistics, writing across the curriculum.

\section{INTRODUCCIÓN}

La universidad, como espacio de formación de nuevos profesionales, no solo tiene responsabilidad sobre la transmisión de conocimientos teóricos y aplicados sino sobre la preparación de los estudiantes para la acción futura en esferas sociales que implican la producción de nuevos conocimientos y la transformación de procesos sociales y tecnológicos. En este sentido, constituye un desafío fundamental para las instituciones de educación superior poner en práctica propuestas pedagógicas que se centren en el objetivo de ampliar la posibilidad de actuación de los sujetos en esos ámbitos. Por otra parte, especialmente en un contexto de democratización de los estudios superiores, es necesario ofrecer herramientas para un mejor desempeño de los estudiantes en el curso de una carrera.

Desde una posición teórica que entiende el aprendizaje como la expansión del potencial de significación (Halliday, 1993) y las diferentes actividades sociales como géneros que implican construcciones discursivas particulares (Martin \& Rose, 2008), la enseñanza de la lectura y la escritura de géneros académicos y profesionales se vuelve central en este contexto. En términos de Bajtín (1985), una nueva esfera de actividad humana propondrá a quienes se inicien en ella el desafío de nuevos géneros discursivos para abordar. $Y$ esos nuevos géneros implican un nuevo uso de la lengua, que debe ser aprendido por los alumnos.

En el ámbito internacional, diferentes propuestas elaboradas en marcos teóricos diversos han dado lugar a una larga tradición de la enseñanza de lectura y escritura basada en el concepto de género (Swales, 1990; Mc. Leod \& Soven, 1992; Hyon, 1996; Marinkovich \& Morán, 1998; Hyland, 2002; Karwoski, Gaydeczka \& Brito, 2006; Bazerman, Bonini \& Figueiredo, 2009). Del mismo modo, en la Argentina se desarrollan cursos y talleres que atienden a la necesidad de 
enseñar géneros propios de la actividad universitaria, especialmente al inicio de los estudios superiores (UNLu, 2001; Pereira \& Di Stefano, 2001; Uslenghi, Padilla \& Singstad, 2002; Carlino, 2006; Padilla, 2007). Otra línea de trabajo es la que considera la necesidad de formar a los estudiantes como miembros de una comunidad disciplinar, en la que los profesores de las diferentes disciplinas se hacen cargo, en el marco del dictado de su materia, de la enseñanza de la lectura y la escritura (UNLu, 2001; Carlino, 2005).

En la Universidad Nacional de General Sarmiento (UNGS), ubicada en el conurbano de la Ciudad de Buenos Aires, la enseñanza de la lectura y la escritura es iniciada en dos espacios curriculares independientes: uno como requisito para ingresar a la universidad (Pereira, 2006; López Casanova, 2009) y otro en el primer año de todas las carreras (Adelstein \& Kugel, 2005). El primero, basado en los conceptos de género y tipos textuales, se propone el abordaje de aspectos lingüísticos formales en textos de divulgación sobre discusiones relevantes en el marco de las disciplinas científicas. El segundo, basado en el modelo multinivel de la lingüística textual, propone la lectura de artículos científicos de diferentes disciplinas, a fin de que los alumnos puedan abordarlos a partir del reconocimiento de su estructura, su función y algunos rasgos lingüísticos prototípicos.

En todas estas aplicaciones, sin embargo, se ha hecho escasa o ninguna mención a la enseñanza, desde una perspectiva funcional, de los usos específicos de recursos lingüísticos y discursivos para la construcción de significado en los diferentes grupos de disciplinas, necesidad sobre la que también llama fuertemente la atención Parodi (2005). Este hecho constituye una limitación de estas experiencias pues, como señalan Halliday y Martin (1993), son los recursos lingüísticos y discursivos los que construyen el conocimiento y las fundaciones ideológicas en que las prácticas científicas descansan. La combinación de rasgos específicos de la lengua de la ciencia hacen posible el discurso teórico, activamente involucrado en la producción de las estructuras conceptuales, de manera que una teoría científica es una construcción lingüística de la experiencia diferente de la que construye la gramática del discurso cotidiano. Si los estudiantes no logran acceder a su dominio no podrán tampoco comprender esas construcciones particulares de mundo que son las disciplinas científicas. Más aun, la lengua de la ciencia se ha extendido a otros ámbitos, como el burocrático o el de los medios, de modo que es imprescindible su aprendizaje para la participación como ciudadanos y debe, entonces, ser enseñada.

Dado que los estudiantes no reciben formación en este sentido en ninguno de los niveles educativos que transitan, ha sido necesario generar en la UNGS otros espacios para la promoción de habilidades de lectura y escritura especializadas, frente a la demanda de estudiantes, profesores y responsables de las carreras. Así, fue diseñado e implementado un programa que se propone enseñar no solo la estructura y contextualización de los nuevos géneros que los 
estudiantes deben abordar, sino las características discursivas que en las diferentes disciplinas se han desarrollado para la construcción de conocimiento. Este programa está basado en los conceptos de género, registro, discurso y lenguaje de la Lingüística Sistémico-Funcional (LSF) (Halliday \& Matthiessen, 2004; Martin, 1992; Martin \& Rose, 2007; Martin \& Rose, 2008).

Esta teoría, que concibe la lengua como un sistema complejo organizado en diferentes niveles o estratos en relación de realización, permite dar cuenta del significado que se construye en un texto y de cómo se lo construye mediante el análisis en todos los niveles, poniendo de relieve la redundancia entre ellos. Asimismo, el modelo estratificado de contexto permite explorar las relaciones entre género y registro y su realización mediante el discurso, así como la identificación y organización en sistemas de los diferentes géneros en un ámbito determinado de la cultura. Además, este marco provee de una caracterización del discurso de las disciplinas (Halliday \& Martin, 1993; Martin \& Veel, 1998; Wignell, 2007), así como de una teoría sobre el rol de la lengua en la construcción de conocimiento (Christie \& Martin, 2007).

Se asume también la propuesta pedagógica desarrollada por la Escuela de Sydney (Martin, 1999) con algunas modificaciones (Moyano, 2007), que reconoce influencia de Bernstein en cuanto la enseñanza explícita y a presupuestos esenciales relativos a la redistribución de poder mediante el acceso a los recursos discursivos. Esta manera de trabajar en las aulas no se centra en la caracterización formal de los géneros ni de rasgos del discurso ni tampoco en la enseñanza sobre aspectos teóricos de la lengua sino en cómo los textos construyen significado relevante en un ámbito social determinado y permite, mediante reflexión metalingüística y metacognitiva explícita, el desarrollo de habilidades discursivas en prácticas de lectura y escritura.

\section{El Programa de Desarrollo de Habilidades de Lectura y Escritura a lo largo de la Carrera (PRODEAC)}

El PRODEAC fue diseñado en un largo proceso institucional y recibió aprobación del Consejo Superior de la UNGS con asignación de presupuesto recurrente en 20051. Su principal objetivo pedagógico es favorecer el desempeño académico de los estudiantes en el curso de sus estudios universitarios y su preparación para las actividades profesionales que realizarán en el futuro. Dado que la construcción de discurso es central en este tipo de actividades, cobra especial relevancia la promoción del desarrollo de habilidades de lectura y escritura especializadas.

Representantes de las diferentes líneas de enseñanza de los géneros académicos señalan la necesidad de que miembros más expertos de las comunidades disciplinares introduzcan o inicien a los estudiantes en las prácticas propias del ámbito. Esta tarea está necesariamente vinculada 
con el trabajo a realizar con los textos escritos mediante los cuales se realizan esas actividades, lo que demanda un conocimiento del experto sobre aspectos que pueden ser iluminados desde el análisis del discurso basado en la lingüística, aporte que puede realizar un profesor de letras ${ }^{2}$. Más que considerar, entonces, la opción entre uno u otro tipo de profesional para llevar a cabo esta tarea ${ }^{3}$, se propone asignar esta responsabilidad a un equipo formado por ambos, en un dispositivo que se dio en llamar 'negociación entre pares', al que cada especialista aporta diferentes saberes que se complementan en beneficio del proceso de enseñanza. El trabajo conjunto supone compartir la responsabilidad de planificación, asignación y evaluación de las tareas escritas propuestas a los alumnos y la aplicación de la propuesta pedagógica en cuyo marco se toman decisiones. Este trabajo conjunto es el que permite favorecer el desarrollo de una conciencia lingüística y genérica en los profesores de las materias específicas, a la vez que aporta al profesor de letras conocimiento del contexto y de los campos disciplinar y profesional puestos en juego, lo que resulta en un incremento de la formación de los participantes en el marco de la interacción en áreas de conocimiento que han sido ajenas a su trayectoria. A lo largo de la aplicación, esta interacción mejora la capacidad de los profesores de materias específicas para orientar el trabajo de escritura.

Por otra parte, la implementación de la enseñanza de lectura y escritura al interior de los espacios curriculares resuelve algunas dificultades que plantean los talleres paralelos. Hyland (2002) señala que la tarea de escritura aparece en ellos separada de la actividad que le da origen y se torna un trabajo de 'composición', con especial acento en los aspectos formales de un tipo de texto. El trabajo en las materias, en cambio, supone el diseño de actividades pertinentes a la instancia de formación, es decir, actividades que respondan a las exigencias específicas del contexto en que se realizan.

Cabe señalar, además, que dada la cantidad de materias que cada carrera incluye en Argentina, no parece posible pensar en añadir cursos obligatorios para la graduación. En cuanto a los talleres optativos, no son adecuados al menos por dos razones. Por un lado, no son solo algunos estudiantes los que requieren formación en esta área ${ }^{4}$, de manera que no resultaría satisfactorio que la opción dependiera de la mera voluntad de los interesados. Por otro, muchos de los estudiantes carecen de disponibilidad horaria para añadir actividades a su carrera universitaria, pues distribuyen su tiempo entre estudio y trabajo. Relegar un aprendizaje de tal importancia a la opción implicaría, una vez más, establecer diferencias en la formación según la posición económico-social de cada sujeto.

Para la implementación de un programa universitario de escritura se ha reconocido como factor fundamental el respaldo institucional (UNLu, 2001; Carlino, 2005; Bazerman, 2007). En el caso que nos ocupa, la elevada dificultad de aplicación de un trabajo conjunto entre profesores de 
dos áreas distintas sin incrementar horas en el currículum, ha requerido de un fuerte compromiso no solo de los actores inmediatos sino de funcionarios de mayor responsabilidad en el rol de agentes organizadores del trabajo en cada carrera. Este respaldo ha asegurado además el financiamiento necesario, lo que permite hacer extensivo el Programa al conjunto de los estudiantes del Ciclo Superior como parte de la formación del perfil de egresado.

A fin de lograr sus objetivos, el PRODEAC se ha organizado en actividades recurrentes. Antes de comenzar cada semestre, se seleccionan las materias de cada carrera en las que se intervendrá. Estas decisiones se toman con los responsables del Área de Formación Académica de cada unidad a cargo del dictado de las carreras, que utilizan criterios particulares en cada caso ${ }^{5}$. Una vez asignadas las materias, se constituyen los equipos de trabajo para cada una, integrados por el o los profesores a cargo y un profesor de letras, que se reúnen periódicamente a lo largo del semestre de aplicación para planificar, monitorear y ajustar el proceso. Los profesores de letras participan de las clases destinadas al trabajo específico con la escritura.

\subsection{La negociación entre pares o 'socios'}

Se ha dado en llamar 'negociación' a la interacción entre un lingüista/profesor de letras del Programa (a partir de ahora, profesor PRODEAC) y el profesor de la materia participante. Se trata de un intercambio entre pares que se reúnen para un trabajo compartido, al que cada uno aporta diferentes saberes y experiencias.

El profesor de la materia específica aporta a la negociación su conocimiento del campo: el referido al dominio disciplinar en cuestión, así como el de las actividades académicas y profesionales que giran en torno de esos saberes. En cuanto al grado de conciencia alcanzado sobre el uso del lenguaje (conciencia lingüística) y sobre los géneros característicos del ámbito académico y profesional (conciencia genérica), se han registrado diferencias entre los profesores con los que se ha trabajado en el Programa (Natale \& Moyano, 2006; Natale, 2007; Moyano, 2009). Por otro lado, estos actores no suelen tener conciencia del discurso como constitutivo de la actividad social, de manera que muchas veces consideran que la reflexión sobre los textos resulta completamente ajena al programa de una materia ligada a la actividad profesional (Valente, Cusolito, Martínez, Abrevaya \& Moyano, 2008).

Por su parte, el profesor PRODEAC, que carece del conocimiento específico de las disciplinas así como de las prácticas profesionales en que se enmarcan los géneros en cuestión, debe tener formación para la descripción de géneros a partir del análisis lingüístico, discursivo y contextual que permite seleccionar los contenidos a enseñar, así como con un cierto grado de 
especialización en el discurso de las ciencias: conocimiento sobre el contexto en el que los textos académicos y científicos se generan y circulan así como sobre sus características, tanto en lo que refiere a la estructura de los diferentes géneros (Swales, 1990; Bathia, 1993; Cubo de Severino, 2005), como a las particularidades del lenguaje típico de esta actividad (Halliday \& Martin, 1993; Wignell, 2007). Dispone, además, de estrategias pedagógicas para la enseñanza de la lectura y la escritura provistas por el Programa.

Durante la negociación, llevada a cabo antes de iniciar el trabajo con los estudiantes, es necesario establecer una serie de acuerdos pedagógicos entre las figuras docentes que interactuarán en el curso afectado al Programa. Esos acuerdos giran en torno a las cuestiones expuestas a continuación.

- 'Función de las actividades de escritura en el proceso de aprendizaje de los contenidos de la materia'. En este punto, es necesario que el docente de la disciplina considere el trabajo de escritura como una tarea integrada a lo que habitualmente se entiende por aprendizaje de contenidos, teniendo en cuenta, por un lado, la función del lenguaje y de la escritura en ese proceso y asociando esta función a la realización de actividades disciplinares o profesionales, por el otro. La experiencia en la implementación del Programa indica que mientras el trabajo sea considerado 'adicional' o 'formal' por los profesores o los estudiantes, no se lograrán los objetivos perseguidos.

- 'Planificación conjunta de las actividades de lectura y escritura que el estudiante llevará a cabo a lo largo de cada materia'. Esta tarea debe realizarse a partir del criterio del profesor a cargo, pero teniendo en cuenta los aportes que el profesor PRODEAC puede hacer acerca de, por ejemplo, la graduación de la dificultad de los géneros y otras cuestiones pedagógicas relativas a la escritura o a la formulación de consignas para tal propósito.

- 'Definición y caracterización de los géneros que los estudiantes deberán producir, incluyendo su contextualización, estructura y rasgos discursivos y lingüísticos característicos'. Es esta una de las instancias claves en el proceso de la negociación. Según Martin y Rose (2008), el trabajo del lingüista en el marco de la enseñanza de géneros consiste en detectar los que resultan relevantes en un área de la cultura y describirlos en todos los estratos de lengua y contexto. En la negociación entre socios, el profesor de la materia, como miembro de la comunidad disciplinar o profesional en la que se pretende formar al estudiante, es quien selecciona el género que pedirá a los estudiantes que produzcan, así como ejemplares textuales que el profesor PRODEAC debe analizar para negociar luego con los estudiantes.

A partir de su conocimiento del campo, el profesor de la materia puede colaborar con la construcción de información acerca del contexto de cultura, especialmente cuando se trata de 
contextos profesionales: quiénes producen los textos en cuestión, en qué condiciones, quiénes son sus destinatarios, cómo se relacionan estos participantes, qué tipo de actividad se realiza mediante el texto, cuáles son los propósitos que se persiguen. Esta información contribuye a controlar las interpretaciones que el lingüista haga a partir del análisis de los textos y, en lo pedagógico, ofrece al estudiante un marco situacional de referencia.

El trabajo del profesor PRODEAC en este punto consiste en analizar los ejemplares textuales que el profesor de la materia aporta, seleccionados de publicaciones prestigiosas y de consulta habitual en la comunidad de referencia. Algunos de los géneros seleccionados están pautados en cuanto a sus características formales en revistas especializadas o formularios institucionales a fin de orientar acerca de la organización de contenidos. Estas pautas no parecen ser suficientes, sin embargo, para quienes tienen que producirlos por primera vez. Se deben caracterizar, entonces, no solo la estructura del género y la función de sus partes, sino también la organización discursiva de cada parte y algunos aspectos lingüísticos, especialmente cuando se trata de géneros poco estudiados. De ahí se seleccionan los rasgos más significativos para su enseñanza en cada momento, incluyendo la introducción de recursos multimodales (gráficos, figuras, esquemas, etc.), sus características, su función, su relación con el texto verbal. Esta selección implica que el Programa irá graduando en lo posible la enseñanza de características del tipo de discurso que sean relevantes y transferibles entre géneros.

En algunos casos, no fue posible contar con textos para el análisis antes de la primera intervención. El trabajo consistió, entonces, en discutir en el marco de la negociación entre socios la propuesta de una estructura para el texto que se solicitaría a los estudiantes. Generalmente, este tipo de trabajo ha permitido que, en el segundo semestre de intervención en la misma materia, el profesor proporcionara los ejemplares necesarios, lo que puede interpretarse como una evolución en términos de conciencia genérica. Otro problema consiste en que muchos géneros profesionales dan lugar a documentos confidenciales, de manera que no se tiene acceso a ellos. Nuevamente, es la negociación entre socios el dispositivo que permite una aproximación a su descripción, en la que el lingüista debe poner en juego su capacidad mediante un abordaje teórico que supone ciertas regularidades en la construcción discursiva en un ámbito específico de la cultura.

- 'Planificación de las intervenciones de los docentes de lengua en las clases durante el dictado de la materia'. No deja de ser problemática la decisión sobre el tiempo de clase que se asignará a la enseñanza de la escritura, actividad que tiene que ser acotada pero suficiente y en relación con la consecución de los objetivos de la materia en el marco más general de la carrera. Una cuestión central es la selección de los contenidos a enseñar en relación con la caracterización del género y el discurso en el ámbito específico, así como el modo en que se 
aplicará el diseño pedagógico en cada caso particular. En este punto, la responsabilidad de la propuesta a negociar debe recaer sobre el profesor PRODEAC.

- 'Elaboración conjunta de pautas de evaluación de los escritos, que permitan dar cuenta del progreso en el aprendizaje de la disciplina y de las habilidades discursivas'. Esta fase del trabajo de negociación suele presentar divergencias en los puntos de vista. Los profesores de las materias tienden a considerar -dada la experiencia transitada en los espacios de su propia formación- que los rasgos a tener en cuenta son formales, fundamentalmente de tipo normativo y gráficos. Pero desde la perspectiva teórica asumida por el PRODEAC se debe atender a aspectos funcionales del texto y, por lo tanto, relativos a la construcción del registro y el género.

Es necesario también negociar las expectativas en cuanto al grado de proximidad o distancia con los textos de expertos, a fin de controlar el proceso de evaluación. Este trabajo -todavía escasamente alcanzado- supone la elaboración de rúbricas que definan las expectativas en tres aspectos: el uso de la información teórica o tecnológica para el trabajo de aplicación, la adecuación del texto a la estructura del género y los rasgos gramaticales y discursivos esperables. Estas expectativas tendrán que graduarse en las diferentes aplicaciones, de manera de evaluar solo aquello que haya sido objeto de enseñanza explícita.

Como con toda herramienta de este tipo aplicada a la evaluación de la escritura, se corre el riesgo de considerar los rasgos deseables establecidos en las rúbricas como normas. En la tarea de enseñanza habrán de tomarse las precauciones necesarias para que esto no ocurra, especialmente porque en el marco teórico seleccionado los géneros son vistos como actividades sujetas a variación diacrónica y sincrónica, y las características discursivas y lingüísticas como rasgos con alto grado de frecuencia de aparición en las instanciaciones, como recursos, no como normas.

Para avanzar en este sentido, hasta el momento se están llevando a cabo trabajos de diagnóstico y seguimiento de los estudiantes, que permitirán establecer no solo diferencias con los de expertos sino la evolución que logran mediante el proceso de enseñanza (Giudice, Natale \& Stagnaro, 2008; Giudice \& Moyano, 2009; Giudice, 2009a; entre otros, en preparación en el marco de la investigación desarrollada en el Programa).

El dispositivo 'negociación entre socios' ofrece, en definitiva, un espacio para discusiones y acuerdos sobre la enseñanza no tradicional de habilidades de lectura y escritura. La lectura y la escritura son vistas en este Programa como la llave para el aprendizaje del conocimiento construido en una disciplina a través de sus textos, así como de las prácticas sociales propias de los ámbitos de desempeño actual y futuro de los estudiantes; en síntesis, como actividades 
que habilitan para la participación social en espacios institucionales. Este trabajo será, entonces, la base de un proceso de enseñanza que amplíe la experiencia y la competencia cultural de los estudiantes en tanto aprendan a reconocer y realizar un rango mayor de contextos, o -dicho en términos de la teoría de códigos socio-semióticos de Bernstein en su diálogo con la LSF (Christie, 1999)- modifiquen la orientación de código para volverla más elaborada, expandiendo el potencial de significación y participación social desarrollados hasta el momento en que el proceso tiene lugar.

\subsection{La enseñanza de los géneros académicos, científicos y profesionales}

En la fase de enseñanza, el profesor PRODEAC adapta a cada situación y al nivel universitario un modelo pedagógico para la enseñanza de la lectura y la escritura (Figura 1) que, como ya se ha dicho, es una adaptación del propuesto por la Escuela de Sydney (Martin, 1999). El modelo propone una secuencia de pasos para la enseñanza que supone la lectura como punto de partida para el aprendizaje de la escritura, el diseño del texto como recurso crítico para la organización de la tarea y de su producto y la edición como habilidad de todo escritor experto. La secuencia se propone, además, un desarrollo progresivo de la autonomía del estudiante en las tareas de lectura y escritura críticas que favorecen el dominio de los géneros disponibles así como la posibilidad de crear variaciones según las necesidades de su actuación. Esta autonomía se logrará a partir de un trabajo conjunto entre el docente y el grupo de estudiantes que gradualmente transitará hacia el trabajo individual. El docente guía la tarea y procura promover el aprendizaje mediante el modelaje de la lectura y la escritura como enseñanza explícita, así como también estimula la colaboración entre alumnos en pequeños grupos para que el trabajo independiente se alcance luego de la experiencia compartida. 


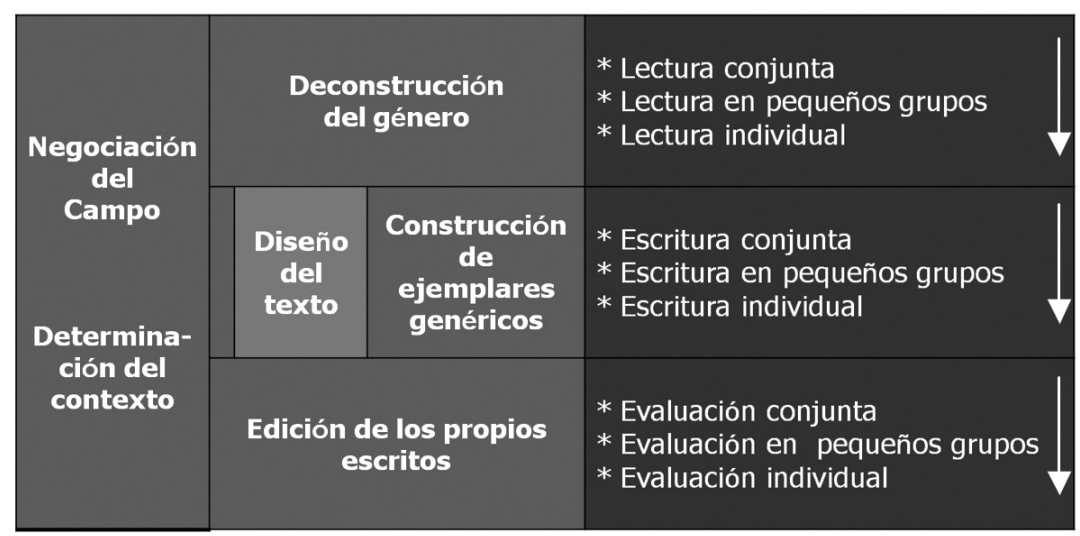

Figura 1. Modelo pedagógico para la enseñanza de la lectura y la escritura (Moyano, 2007).

La negociación del campo y la determinación del contexto son momentos centrales de la propuesta, que atraviesan todo el proceso de enseñanza. La negociación del campo está a cargo del profesor de la materia en lo que se refiere a la enseñanza de los contenidos específicos del espacio curricular. Ingresan también a la discusión las actividades académicas o profesionales que se llevan a cabo a través del texto, cuestión que abordan ambos socios de enseñanza en su trabajo de descripción del género o el profesor PRODEAC hasta lograr el dictado conjunto de clases. La determinación del contexto implica discutir la función que en una cultura específica tiene el género en cuestión, qué roles juegan quienes intervienen en la actividad social que supone y sus relaciones con otros géneros propios del ámbito en discusión.

Es importante destacar que entre todas las corrientes teóricas que se ocupan de la enseñanza de los textos científicos y académicos existe consenso acerca de la necesidad de reflexionar sobre el contexto con los estudiantes, ya sea a fin de que adquieran conciencia de las opciones retóricas disponibles que motivarán las selecciones lingüísticas (Hyon, 1996; Bazerman, 1988) o a fin de hacer significativa la producción escrita en contextos de clase (Hyland, 2002). En el marco seleccionado por el programa, la reflexión se orienta a vincular los textos con el contexto cultural y con los propósitos que los orientan, así como una primera identificación de la configuración de campo, tenor y modo en juego (Martin \& Rose, 2008).

La fase de deconstrucción sigue a ese primer momento, y consiste en identificar junto con los estudiantes la estructura del género y aquellos aspectos de discurso y léxico-gramática que el docente PRODEAC haya seleccionado para la intervención. Nuevamente, el trabajo conjunto de 
los socios de enseñanza resulta deseable en este momento del proceso. Cómo se estructuran los textos en cada género, cómo suele estar organizada la información en los diferentes pasos de la estructura, su función y las relaciones entre ellos son contenidos a enseñar. En los casos en los que no se hubiera obtenido un ejemplar para deconstruir -cosa que ya se ha señalado ocurre con frecuencia en el primer semestre de aplicación en una materia-, los profesores PRODEAC orientan a los estudiantes en la elaboración conjunta de un esquema de la estructura del género, siguiendo los acuerdos que se hubieran podido establecer entre los socios de enseñanza.

Cuando los estudiantes logran organizar sus trabajos escritos de la manera esperada -objetivo que demanda un importante trabajo de interacción en el aula- han recorrido buena parte del camino hacia la apropiación del género en cuestión. Sin embargo, es imprescindible reflexionar también sobre los recursos discursivos y lingüísticos utilizados en el texto que se deconstruye, dado que muchos de los problemas de escritura de los estudiantes se dan en este nivel. El trabajo se propone como modo de promover la toma de conciencia de las selecciones de los escritores a partir de un conjunto de opciones para la construcción de significados en las tres metafunciones del lenguaje y su contribución a la construcción del registro. Así, el estudiante aumenta la comprensión del campo no solo en cuanto a contenidos sino en cuanto a la construcción de la disciplina en juego como espacio de diálogo entre diferentes posiciones.

En esta instancia, entonces, los estudiantes desarrollan habilidades de lectura y de aprendizaje de la materia en cuanto adquieren estrategias de abordaje de los textos bajo la guía del docente. Por otra parte, obtienen un cuadro relativamente claro de la producción que de ellos se espera. Pero la deconstrucción, aunque esencial, no resulta suficiente en sí misma para la enseñanza de la escritura, sino que requiere del cumplimento de los siguientes pasos del diseño pedagógico.

En la adaptación del modelo a la educación superior, la etapa de escritura conjunta ha quedado hasta el momento eliminada, a excepción de que en la clase se introduzcan rasgos específicos de lengua y discurso de las disciplinas, cuyo aprendizaje requiere este tipo de trabajo. Es fundamental, en cambio, negociar en las clases con los estudiantes el diseño de los textos a producir. El trabajo de interacción alrededor de esquemas previos a la escritura suele ser un trabajo de suma utilidad para los estudiantes, especialmente cuando tienen que producir textos en el marco de géneros no frecuentados antes por ellos (Moyano, 2004b, Giudice, 2009b) o cuando no están habituados a operar con grandes cantidades de información para organizar. Por otra parte, esta dificultad ha sido también detectada en estudiantes de postgrado así como en investigadores en experiencias anteriores (Moyano, 2000) y la discusión grupal de diseños textuales se ha revelado también en ese contexto como una estrategia con resultados positivos. 
Como se observa en la Figura 1, el diseño pedagógico contempla la existencia de una fase denominada edición de los textos, que supone al inicio un trabajo conjunto. Este dispositivo, más que una 'corrección' en términos tradicionales, implica una nueva instancia de aprendizaje, en la que se orienta al estudiante a la realización de un paso de la producción de escritos habitual para los expertos. Para realizar este trabajo, se deconstruye en interacción entre el profesor y el grupo un texto producido por alguno de los estudiantes, que se considera como ejemplar de un género que construye un campo específico. Así, se lo ubica en su contexto situacional y de cultura, para considerar desde esa perspectiva si las selecciones lingüísticas y discursivas han sido adecuadas, teniendo en cuenta las opciones que el sistema ofrece, y si la estructura esquemática esperable fue lograda en el texto, de acuerdo con las expectativas sociales de realización del género como actividad, cuidando especialmente los aspectos funcionales (Eggins, 1994). En este proceso, los estudiantes señalarán lo que consideren mejorable en el texto y se discutirán en conjunto las alternativas posibles. En el espíritu del modelo, la tarea se orienta a ofrecer herramientas a los estudiantes para que adquieran progresivamente autonomía en esta etapa crítica de la escritura y que realicen aprendizajes transferibles.

No siempre se ha podido implementar en el marco del Programa la edición conjunta de los textos. Por un lado, esta práctica se ve poco favorecida por el tiempo disponible frente a los estudiantes; por otro, prevalecen en los profesores las prácticas más tradicionales, en la convicción de que la corrección individual de cada escrito por el docente de lengua es de mayor provecho para los estudiantes. Sin embargo, un trabajo de este tipo, que podría denominarse 'edición asistida', no se constituye en una instancia de aprendizaje ni favorece la autonomía.

La edición asistida se diferencia de la edición conjunta en el grado de participación de los estudiantes y el profesor en la propuesta de modificación del texto a editar. En la edición asistida, es el profesor el que identifica problemas en el texto de cada alumno y sugiere las modificaciones que considera necesarias. En la edición conjunta, todo el grupo evalúa el texto de un estudiante en interacción con el docente para juzgar su adecuación e identificar los aspectos que generen problemas para la construcción de sentido. Asimismo, todo el grupo propone modificaciones para resolver los problemas detectados, propuestas que son discutidas en la clase antes de ser aceptadas o rechazadas. De esta manera, se logra modelar la tarea de edición con la guía del profesor, a fin de que luego, mediante nuevas experiencias conjuntas o en pequeños grupos de andamiaje entre pares, el estudiante pueda hacer la experiencia de edición individual con buenos resultados.

Cabe señalar, además, que la corrección tradicional o edición asistida demanda un tiempo excesivo a los docentes del Programa, especialmente si se aplica la práctica de escritura y re-escritura con evaluación. En gran cantidad de casos, esta tarea se ha realizado a través del 
correo electrónico (Stagnaro, Natale \& Moyano, 2008), lo que significa para los docentes una importante carga de trabajo y, como consecuencia, un alto costo institucional, considerando que cada profesor es responsable de la asistencia de varias materias y que cada estudiante presenta más de un escrito de entre por lo menos 15 y 20 páginas por semestre.

En experiencias realizadas en cursos de escritura al inicio de la carrera, la aplicación de la estrategia que implica el tránsito de la edición conjunta a la individual produjo resultados destacados, no solo en el mejoramiento de los textos sino en la adquisición de autonomía por parte de los estudiantes (Moyano, 2007) ${ }^{6}$. Es necesario, entonces, intensificar y extender esta práctica en el PRODEAC, planificando cuidadosamente su implementación a fin de que el costo del Programa medido en tiempo de afectación por profesor se vea reducido y que, al mismo tiempo, los estudiantes se propongan como objetivo alcanzar cada vez mayor autonomía.

Como dispositivo adicional, a pedido de algunos responsables académicos, se incluyó en el Programa la implementación de lo que suele conocerse como 'laboratorio de escritura', esto es, un espacio en el que los estudiantes reciben asesoramiento para la producción de los textos solicitados por sus profesores. En el caso del PRODEAC, este ha sido un espacio de 'refuerzo' de la enseñanza, en términos tradicionales. La asistencia era voluntaria, a fin de hacer consultas en cualquier momento del procesamiento del texto. Tal como la edición asistida, el recurso resultó de alto costo en tiempo de los profesores y no produjo los resultados buscados a excepción de algún caso aislado.

Por otra parte, los estudiantes no siempre acudieron al laboratorio, salvo por mediación del profesor de la materia. Cuando el dispositivo sustituyó el trabajo de negociación entre pares, no se registró asistencia de los estudiantes. Es posible afirmar, entonces, que cuando el profesor de la disciplina no participa del trabajo conjunto, esta actitud es interpretada por el estudiante como una señal de que el aprendizaje de la escritura académica o profesional y el de la disciplina son independientes y tienen diferentes jerarquías.

A luz de estas experiencias, se decidió suspender el laboratorio y fortalecer la aplicación de las estrategias diseñadas para el intercambio en el aula. Sin embargo, es posible pensar en mantenerlo con modificaciones, a fin de que ofrezca un espacio para trabajar de manera más sistemática aspectos relacionados con el lenguaje de las disciplinas, una vez que estos se hayan tratado en las clases, a sabiendas de que puede reproducir el trabajo en talleres que en su concepción el Programa ha dejado de lado. Por eso es necesario remarcar que el proceso debe realizarse siguiendo el modelo pedagógico diseñado para su aplicación a grupos, procurando evitar la enseñanza individual. Será necesario también estudiar la manera de alentar la asistencia de los estudiantes por derivación de los profesores de la materia, lo que requerirá de tra- 
bajo específico en el marco de la negociación. Finalmente, teniendo en cuenta las ventajas del trabajo a distancia, se podrá estudiar la posibilidad de sostenerlo en plataformas de enseñanza electrónica, a condición de que admitan el andamiaje entre pares en actividades grupales.

\section{Evaluación de los logros alcanzados}

Los logros obtenidos en el marco del PRODEAC, luego de 5 años a partir de su creación, se relacionan con diferentes aspectos críticos: inserción institucional, cumplimiento de los objetivos pedagógicos, desarrollo de aprendizajes de los actores involucrados, evaluación y mejora del diseño en cada una de sus fases y globalmente, producción de información en investigación.

Las condiciones de aplicación en la UNGS han permitido, como logro institucional, que el PRODEAC fuera expandiendo su campo de aplicación de 6 materias en el primer semestre de implementación hasta la atención de entre 14 y 20 materias por semestre en las aplicaciones más recientes, abarcando todas las carreras dictadas a excepción de la de creación más reciente. El compromiso institucional determinó no solo un funcionamiento adecuado que en cada Instituto de la Universidad adquiere características especiales sino la creación de nuevos cargos para la inserción de investigadores-docentes de planta con afectación al Programa. Este proceso llevó el número inicial de 2 a 7 en 5 años. Esta evolución permitió también la conformación de un grupo que distribuye su tiempo entre la práctica pedagógica y la investigación a fin de monitorear permanentemente el Programa y desarrollar estrategias que contribuyan al mejoramiento de la intervención, además de producir conocimiento específico sobre el objeto de enseñanza. Algunos de estos desarrollos han merecido nuevos proyectos de investigación, ya sea en el ámbito de la UNGS, en colaboración con universidades nacionales y extranjeras, o en tesis doctorales. Finalmente, para favorecer la comunicación de lo realizado y con el fin de ir generando un espacio de interacción virtual, se construyó el sitio web del Programa, que ya está disponible ${ }^{7}$.

Los estudiantes muestran una evolución en el desarrollo de habilidades de escritura, detectado a través de sus textos. Este seguimiento ha puesto en evidencia un avance en la habilidad de estructurar los escritos según lo esperable para el género y un progreso en la construcción discursiva. Si bien gran parte de la información producida se basa en instanciaciones de número reducido por alumno, se ha podido observar a partir de su seguimiento en más de un semestre que los estudiantes desarrollan habilidades que transfieren a nuevas situaciones (Giudice et al., 2008; Giudice, 2009a, 2009b). Asimismo, son capaces de hacer demandas que denotan un incremento en la conciencia lingüística, a partir de un primer reconocimiento intuitivo de la diferencia entre el discurso de expertos y el que ellos mismos producen. 
El logro de la autonomía en la escritura es una tarea difícil, pero los estudiantes tienden a mejorar también en este aspecto a medida que transitan la carrera y se acercan a la graduación. Por otro lado, existe un incremento en el compromiso de los estudiantes en este proceso, que se pone de manifiesto a partir de la asistencia a las clases de intervención en la materia, las consultas realizadas y el trabajo en relación con la escritura.

Los docentes a cargo de las materias evolucionan en cuanto al desarrollo de conciencia lingüística y genérica a lo largo de su trabajo en el Programa, efecto que se atribuye al espacio de negociación entre pares. En cuanto a lo pedagógico, no solo llegan a acuerdos muy bien establecidos con sus socios, sino que incrementan el grado de conciencia de la necesidad de la enseñanza de la lectura y la escritura especializadas y van asumiendo autonomía en la toma de decisiones y generación de estrategias para la enseñanza de géneros vinculados a sus materias: se vuelven propositivos, participan de clases conjuntas y contribuyen al mejoramiento del proceso, además de producir cambios en la manera de evaluar los escritos de los estudiantes y en las demandas que hacen al Programa. Esto pone también en evidencia un aumento del compromiso asumido.

Pese a que los integrantes del equipo PRODEAC cuentan con diferente tiempo de experiencia en la tarea, puede decirse que la apropiación de la propuesta se produce cada vez con mayor rapidez. Se ha notado una importante evolución de estos actores en la conducción del proceso de negociación con los socios de enseñanza, al que cada uno aporta propuestas que van enriqueciendo la práctica y el desarrollo del dispositivo. Asimismo, realizan un trabajo diferenciado en la aplicación de cada fase del diseño pedagógico, que se comparte con los demás integrantes del equipo en espacios de discusión. Estas discusiones periódicas redundan en una progresiva mejora de las implementaciones e iluminan los aspectos que requieren mayor investigación. Por otra parte, gracias a la negociación entre socios se ha incrementado el conocimiento de las situaciones que se resuelven mediante la escritura en el ámbito profesional y en algunas prácticas académicas particulares de cada espacio de enseñanza.

Se ha avanzado también en la descripción de géneros y comienza a producirse mayor información sobre las características del discurso en cada paso de la estructura genérica así como en las características de lenguaje en las disciplinas en español a través de la implementación de diferentes investigaciones que se vinculan en el Programa.

\section{El desafío actual}

Hasta el momento, la tarea que se ha realizado en el PRODEAC se ha concentrado, sobre todo, en la enseñanza de la estructura esquemática de los géneros a producir por los estudiantes, 
más la de algunos rasgos lingüísticos, con algún avance en la especificidad del discurso de las disciplinas. Es necesario, sin embargo, avanzar en el diseño de actividades que permitan la enseñanza más sistemática de rasgos léxico-gramaticales y discursivos, atendiendo a los problemas identificados (Giudice \& Moyano, 2009). Si bien hay algunos antecedentes de trabajos en este terreno (Ravelli \& Ellis, 2004), no hay suficiente información disponible acerca de implementaciones de este tipo en el ámbito universitario de grado en español.

Para avanzar en este sentido, tal como lo señala Parodi (2005), es necesario disponer de un conocimiento mayor del objeto de enseñanza, es decir, de mayor investigación sobre diferentes géneros académicos y profesionales en diferentes campos disciplinares en español. Desde su origen, el PRODEAC se propuso generar espacios para avanzar con investigaciones en la caracterización del discurso especializado en español aplicando los desarrollos de la LSF, especialmente los de Martin y Rose $(2003,2007,2008)$ para el análisis de discurso y género y los avances realizados sobre la evolución del discurso de las ciencias y las humanidades (Halliday \& Martin, 1993; Martin, 2007; Wignell, 2007).

Halliday y Martin (1993: 4-12) señalan que la lengua de la ciencia ocupa "un espacio semiótico extendido de límites difusos y variación interna considerable" pero que puede ser definido y reconocido por ciertos "síndromes", definidos como "patrones de co-ocurrencia entre rasgos en los diferentes niveles lingüísticos". En cuanto a la construcción de la experiencia (metafunción ideacional), reconocen la conformación de jerarquías taxonómicas de léxico técnico específico y el uso de ciertos recursos gramaticales, como grupos nominales muy complejos y cláusulas constituidas como relaciones de identificación o causa, por ejemplo, entre procesos nominalizados. Estas cláusulas, además, se combinan a fin de construir formas particulares de razonamiento. Ambos rasgos (uno morfológico y el otro sintáctico) son considerados interdependientes, como diferentes aspectos de un único proceso semiótico: en el nivel léxicogramatical, como síndrome de rasgos de la cláusula; en el semántico, como rasgo del discurso. En el análisis, no interesa tanto reconocer la presencia de estos recursos sino cómo interactúan en el texto para la construcción de significado a fin de hacer interpretaciones en cuanto a la construcción de conocimiento (campo del registro).

Las formas particulares de razonamiento a la que estos autores hacen referencia ponen en juego otros sistemas del discurso, especialmente los que construyen significados textuales. La introducción y el seguimiento de participantes (identificación) suele ser un problema para los estudiantes, de manera que es necesario hacer un trabajo en relación con ello. La periodicidad es -para quienes quieren adquirir habilidades para organizar la información en un texto, particularmente si es extenso y de contenido abstracto- un aspecto crítico: los conceptos de macro e hiper Temas y macro e hiper Nuevo resultan herramientas muy útiles tanto para la 
comprensión como para la producción de textos complejos. Finalmente, reconocer patrones en la construcción del método de desarrollo en las diferentes partes o secciones de los textos en cuestión, así como el reconocimiento de temas en dos capas será otro elemento a tener en cuenta y permitirá entender la interacción con diferentes sistemas, como el de voz, entre otros (Moyano, en prensa).

La valoración en los textos también debe ser objeto de trabajo, particularmente en lo que hace al sistema de involucramiento ${ }^{8}$ a fin de analizar los efectos retóricos de las combinaciones de recursos lingüísticos mediante los cuales un autor asume una postura frente a otras que referencia en el texto, así como también con respecto a su auditorio (Martin \& White, 2005). También interesa analizar cómo funcionan la apreciación, particularmente la construcción de juicio, la gradación y el foco en el discurso de las disciplinas así como en diferentes pasos o secciones de los géneros en cuestión (Hood \& Martin, 2005). Además de otros recursos léxicogramaticales en juego, será de interés atender a algunos Temas interpersonales que tienen una función en este aspecto. Los recursos disponibles en el sistema de valoración permiten construir la figura del autor y su relación con los lectores desde una perspectiva intersubjetiva, de ahí su interés para explorar relaciones tanto en el ámbito de las ciencias como de los espacios profesionales.

La interacción entre estos aspectos del discurso permite la elaboración del campo disciplinar, así como la del tenor, en términos de construcción del autor y negociación de la legitimación del conocimiento construido (Moyano, 2005). De esta manera se aporta a la construcción del género como configuración particular de las tres variables del registro.

Finalmente, es imprescindible diseñar materiales didácticos para el trabajo con los estudiantes en este nivel de formación, de modo que en las próximas aplicaciones del PRODEAC se incremente el trabajo sobre los aspectos discursivos señalados.

\section{Comentarios finales}

En este artículo se ha presentado un programa diseñado con el objetivo de incidir no solo en la evolución adecuada de los estudiantes en el marco de los estudios superiores sino también en su inserción y desarrollo profesionales en el futuro. Estos objetivos, de acuerdo con los lineamientos teóricos y de aplicación de la LSF sobre lengua, cultura, aprendizaje y construcción del conocimiento, tienen una relación directa con el desarrollo de habilidades de lectura y escritura en contextos sociales nuevos para los estudiantes. De ahí su complejidad y la relevancia de su logro, crucial para la inserción social en contextos institucionales. 
El Programa implementado en la UNGS resulta original no solo en sus características de diseño sino en cuanto a que involucra activamente a una serie de actores institucionales de diferentes jerarquías y especialidades, lo que le ha permitido una inserción y un apoyo institucional poco habituales. Esto se ha logrado también gracias a que en sus documentos fundacionales esta universidad reconoce la necesidad de contar con herramientas pedagógicas y especialmente de enseñanza de la lengua que contribuyan a la formación de estudiantes que, en su mayoría, provienen de sectores sociales poco privilegiados (Coraggio, 1994).

En cuanto a los logros obtenidos en 5 años de desarrollo, no solo se destacan los relativos al grado de institucionalización en términos de interés y compromiso de quienes ocupan espacios jerárquicos individuales o colegiados, sino también en lo que se refiere a la construcción del trabajo conjunto de los socios de enseñanza, el progreso en el aprendizaje de los estudiantes, el enriquecimiento del Programa mediante los aprendizajes de sus actores en la experiencia y a través de la reflexión producida en investigación. Cada uno de estos aspectos resulta crítico en sí mismo, pero también en cuanto a su incidencia recíproca.

Como se ha remarcado, existe todavía mucha tarea por realizar: avanzar en la descripción de los géneros y el discurso de las disciplinas en español mediante investigación y producir materiales didácticos para los estudiantes son las que, por el momento, se presentan como urgentes.

\section{REFERENCIAS BIBLIOGRÁFICAS}

Adelstein, A. \& Kuguel, I. (2005). Los textos académicos en el nivel universitario. Los Polvorines: Universidad Nacional de General Sarmiento.

Bajtin, M. (1985). Estética de la creación verbal. Buenos Aires: Siglo XXI.

Bazerman, C. (1988). Shaping written knowledge: The genre and activity of the experimental article in science [en línea]. Disponible en: http://wac.colostate.edu/aw/books/bazerman_shaping/

Bazerman, C., Bonini, A. \& Figueiredo, D. (Eds.). (2009). Genre in a changing world [en línea]. Disponible en: http://wac.colostate.edu/books/genre/\#pub_info

Carlino, P. (2005). Escribir, leer y aprender en la universidad. Una introducción a la alfabetización académica. Buenos Aires: FCE.

Carlino, P. (Ed.). (2006). Procesos y prácticas de escritura en la educación superior. Signo \& Seña $N^{\circ} 16$ [en línea]. Disponible en: http://www.escrituraylectura.com.ar/posgrado/ revistas/SyS16.pdf 
Christie, F. (Ed.). (1999). Pedagogy and the shaping of consciousness. Linguistic and social processes. London: Continuum.

Christie, F. \& Martin, J. R. (2007). Language, knowledge and pedagogy: Functional linguistic and sociological perspectives. London: Continuum.

Coraggio, J. (1994). Reforma pedagógica: Eje de desarrollo de la enseñanza superior. Documentos de Trabajo 1. Estudios de apoyo a la organización de la Universidad Nacional de General Sarmiento. San Miguel: UNGS.

Cubo de Severino, L. (Coord.). (2005). Los textos de la ciencia. Principales clases del discurso académico-científico. Córdoba: Comunicarte.

Eggins, S. (1994). An introduction to Systemic-Functional Linguistics. London: Cassell.

Eggins, S. \& Martin, J. R. (2003). El contexto como género: Una perspectiva lingüístico-funcional. Revista Signos, 36(54), 185-205.

Giudice, J. (2009a). Apropiación por parte de alumnos universitarios de géneros académicos y del tipo de lenguaje propio de las ciencias sociales y humanas: Análisis evolutivo. Ponencia presentada en el V Congreso ALSFAL, Universidad Nacional de Mar del Plata, Argentina.

Giudice, J. (2009b). Implementación del Programa "Desarrollo de habilidades de escritura a lo largo de la carrera" (PRODEAC) en dos materias de la Licenciatura en Economía Industrial: Análisis evolutivo de casos. Ponencia presentada en la Jornada de Intercambio de Experiencias Universitarias en el Desarrollo de Competencias Comunicativas, Universidad Tecnológica Nacional (UTN), Facultad Regional General Pacheco, Argentina.

Giudice, J. \& Moyano, E. (2009). Grado de apropiación del discurso de las ciencias sociales y humanas por alumnos universitarios: Una evaluación diagnóstica. Ponencia presentada en el V Congreso Internacional de la Cátedra Unesco, Instituto Pedagógico de Caracas, Venezuela.

Giudice, J., Natale, L. \& Stagnaro, D. (2008). El proceso de construcción de un estudiante reflexivo. Análisis evolutivo de un caso. Ponencia presentada en el III Congreso Internacional "Transformaciones culturales: Debates de la teoría, la crítica y la lingüística". FFyL Universidad de Buenos Aires, Argentina.

Halliday, M.A.K. (1993). Towards a language-based theory of learning. Linguistics and Education, 5, 93- 116.

Halliday, M.A.K. \& Martin, J. R. (1993). Writing science: Literacy and discursive power. Pittsburgh: University of Pittsburgh Press.

Halliday, M.A.K. \& Matthiessen, C. (2004). An introduction to functional grammar. London: Arnold.

Hood, S. \& Martin, J. R. (2005). Invocación de actitudes: El juego de la gradación de la valoración en el discurso. Revista Signos, 38(58), 195-220. 
Hyland, K. (2002). Teaching and researching writing. London: Longman.

Hyon, S. (1996). Genre in three traditions: Implications for ESL. TESOL Quarterly, 30(4), 693-722.

Karwoski, A., Gaydeczka, B. \& Brito, K. (Org.). (2006). Gêneros textuais: Reflexões e Ensino. Rio de Janeiro: Lucerna.

López Casanova, M. (Coord.). (2009). Los textos y el mundo. Una propuesta integral para talleres de lectura y escritura. Los Polvorines: Universidad Nacional de General Sarmiento.

Marinkovich, J. \& Morán, P. (1998). La escritura a través del currículum. Revista Signos, 31(43), 165-171.

Martin, J. R. (1992). English text: System and structure. Amsterdam: Benjamins.

Martin, J. R. (1999). Mentoring semogenesis: ‘Genre-based' literacy pedagogy. En F. Christie (Ed.), Pedagogy and the shaping of consciousness. Linguistic and social processes (pp. 123-155). London: Continuum.

Martin, J. R. (2007). Construing knowledge: A functional linguistics perspective. En F. Christie (Ed.), Pedagogy and the shaping of consciousness. Linguistic and social processes (pp. 34-64). London: Continuum.

Martin, J. R. \& Veel, R. (Eds.). (1998). Reading science. Critical and functional perspectives on discourses of science. London: Routlege.

Martin, J. R. \& White, P. (2005). The language of evaluation. Appraisal in English. London: Palgrave.

Martin J. R. \& Rose, D. (2007). Working with discourse. Meaning beyond the clause. London: Continuum.

Martin J. R. \& Rose, D. (2008) Genre relations. Mapping culture. London: Equinox.

Mc. Leod, S. \& Soven, M. (Eds.). (1992). Writing across the curriculum: A guide to developing programs [en línea]. Disponible en: http://wac.colostate.edu/aw/books/

Moyano, E. (2000). Comunicar ciencia. El artículo científico y las presentaciones a congresos. Lomas de Zamora: Universidad Nacional de Lomas de Zamora.

Moyano, E. (2004a). La escritura académica: Una tarea interdisciplinaria a lo largo de la currícula universitaria. Revista Texturas, 4(4), 109-120.

Moyano, E. (2004b). La enseñanza de la escritura científico-académica en contextos de pregrado universitario: El caso de la clasificación como micro-género de una entrada de enciclopedia. En S. Hassan \& P. Parra (Eds.), Actas de congreso: "La lingüística sistémicofuncional, la lengua y la educación”. Mendoza: Universidad Nacional de Cuyo.

Moyano, E. (2005). Construcción de conocimiento y su legitimación en la sección Discusión del artículo científico. Ponencia presentada en el VI Congreso de la Asociación Argentina de Semiótica. FFyL Universidad de Buenos Aires, Argentina. 
Moyano, E. (2007). Enseñanza de habilidades discursivas en español en contexto pre-universitario: Una aproximación desde la LSF. Revista Signos, 40 (65), 573-608.

Moyano, E. (2009). Negotiating genre: Lecturer's awareness in genre across the curriculum Project at the university level. En C. Bazerman, A. Bonini \& D. Figueiredo (Eds.), Genre in a changing world (pp. 442-264). Colorado/Indiana: Parlor Press and WAC Clearinghouse.

Moyano, E. (en prensa). El sistema de Tema en español: Una mirada discursiva sobre una cuestión controvertida. En E. Ghio \& M. Fernández (Eds.), Sobre temas de Lingüística Sistémico-Funcional. Santa Fe: Universidad Nacional del Litoral.

Moyano, E. \& Natale, L. (2006). Enseñanza de la lectura y la escritura a lo largo de la carrera universitaria como política institucional: Una investigación acción. Ponencia presentada en el I Congreso Nacional "Leer, Escribir y Hablar Hoy", Universidad Nacional del Centro de la Provincia de Buenos Aires, Argentina.

Natale, L. (2007). ¿Conciencia genérica en profesores universitarios? Ponencia presentada en el III Congreso de la Asociación de Lingüística Sistémico-Funcional de América Latina, Benemérita Universidad Autónoma de Puebla, México.

Natale, L. \& Moyano, E. (2006). Evolución de las conceptualizaciones sobre el lenguaje escrito como herramienta para el aprendizaje en algunos profesores de materias universitarias. En A. M. Rodi \& M. Casco (Coord.), Lengua-Investigación. Actas Primer Congreso Nacional "Leer, Escribir y Hablar Hoy", Universidad Nacional del Centro de la Provincia de Buenos Aires, Argentina.

Padilla, C. (2007). ¿Enseñar a argumentar en la universidad? ¿Por qué y para qué? Actas Primeras Jornadas Latinoamericanas de Lectura y Escritura. Lecturas y escrituras críticas: Perspectivas múltiples [en línea]. Disponible en: http://www.filo.unt.edu.ar/jorn_unesco/ jorn_unesco_cd.htm

Parodi, G. (2005). Discurso especializado e instituciones formadoras: Encuentro entre lingüística e informática. En G. Parodi (Ed.), Discurso especializado e instituciones formadoras (pp. 15-40). Valparaíso: Ediciones Universitarias de Valparaíso.

Pereira, M. \& di Stefano, M. (2001). Diseño curricular del Taller de Lectura y Escritura universitario. La lectura y la escritura como procesos y como prácticas. Ponencia presentada en el XIX ${ }^{\circ}$ Congreso AESLA, Universidad de León, España.

Pereira, M. (Coord.). (2006). La comunicación escrita en el inicio de los estudios superiores. Los Polvorines: Universidad Nacional de General Sarmiento.

Ravelli, L. \& Ellis, R. (Eds.). (2004). Analysing academic writing: Contextualized frameworks. London: Continuum.

Stagnaro, D., Natale, L. \& Moyano, E. (2008). Tutoría vía e-mail: Dispositivo de un programa de enseñanza de escritura académica en lengua materna. Ponencia presentada en el $4^{\circ}$ Congreso ALSFAL, Universidad Federal de Santa Catarina, Florianópolis, Brasil. 
Swales, J. M. (1990). Genre analysis: English in academic and research settings. Cambridge Applied Linguistics. Cambridge:Cambridge University Press.

UNLu, (2001). La lectura y escritura como prácticas académicas universitarias [en línea]. Disponible en: http://www.unlu.edu.ar/ redecom/borrador.htm

Uslenghi, M., Padilla, C. \& Sigstad, M. (Eds.). (2002). Textos de especialidad: Problemas y propuestas para la Universidad. RILL, 15.

Valente, E., Cusolito, F., Ramírez, O., Abrevaya, C. \& Moyano, E. (2008). Un programa de enseñanza de la escritura en las carreras de ingeniería en la UNGS. Análisis de la experiencia con la materia Desarrollo de Productos. Ponencia presentada en el VI Congreso Argentino de Enseñanza de la Ingeniería (CAEDI). Salta, Argentina.

Wignell, P. (2007). On the discourse of social science. Australia: Charles Darwin University Press.

\section{NOTAS}

1 Para una breve historia de este proceso, ver http://www.ungs.edu.ar/prodeac/historia-prodeac. html

2 Cabe señalar que los aquí llamados 'profesores de letras' o 'profesores de lectura y escritura' son en su mayoría investigadores-docentes de la UNGS y muy pocos docentes contratados, también con formación universitaria y desempeño en investigación.

3 Para la discusión generada sobre esta cuestión ver Moyano (2004a).

4 Reconocimientos de esta necesidad resultan la progresiva generalización de cursos de escritura impartidos en las carreras de postgrado o destinados a profesionales e investigadores noveles y el hecho de que organismos de evaluación de las carreras universitarias insistan en la necesidad de incluir la enseñanza de habilidades comunicativas. La especificidad de los géneros en cuestión y su complejidad en cuanto a contenidos teóricos hace que su enseñanza no corresponda a niveles educativos anteriores. Cabe señalar, sin embargo, que una iniciación al discurso de las ciencias es necesaria en los niveles educativos primarios y secundarios, a fin de que los alumnos desarrollen habilidades para comprender y producir textos en los diferentes campos que abordan en la escuela. Esto favorecería también el acceso a la universidad y, probablemente, los talleres al inicio de los estudios superiores.

5 El PRODEAC se aplica en todas las carreras de grado dictadas en la UNGS, a excepción de Cultura y Lenguajes Artísticos, de reciente creación, que cuenta entre sus docentes a numerosos profesores del área de las ciencias del lenguaje. Las carreras de aplicación son Ingeniería Industrial y Electromecánica, Licenciaturas en Economía Industrial y Política, Administración Pública, Políticas Sociales, Educación, Estudios Políticos, Comunicación, Ecología Urbana y Urbanismo y Profesorados de Matemática, Física, Filosofía, Historia y Economía. La distribución de la participación por materias puede observarse en http://www.ungs.edu.ar/prodeac/materias-que-participan-prodeac.html. Cabe señalar que algunas de las materias seleccionadas son compartidas 
por varias carreras, razón por la cual fueron elegidas por los responsables académicos de cada Instituto.

6 Sobre resultados de esta práctica en el PRODEAC, se está trabajando en el marco de la investigación y se cuenta ya con algunos resultados que se preparan para su publicación.

7 http://www.ungs.edu.ar/areas/prodeac_inicio/1/inicio.html

8 El término 'involucramiento' se emplea aquí para traducir 'engagement' al español porque se lo considera más adecuado que el habitualmente usado 'compromiso' para referirse a los 'recursos [lingüísticos] de posicionamiento intersubjetivo’ (Martin \& White, 2005). 